\title{
Comparative study of neutron and nuclear matter with simplified Argonne nucleon-nucleon potentials
}

\author{
M. Baldo, ${ }^{1}$ A. Polls,${ }^{2}$ A. Rios, ${ }^{3}$ H.-J. Schulze, ${ }^{1}$ and I. Vidaña ${ }^{4}$ \\ ${ }^{1}$ INFN Sezione di Catania, Dipartimento di Fisica, Via Santa Sofia 64, I-95123 Catania, Italy \\ ${ }^{2}$ Departament d'Estructura i Constituents de la Matèria, Universitat de Barcelona, E-08028 Barcelona, Spain \\ ${ }^{3}$ Department of Physics, Faculty of Engineering and Physical Sciences, University of Surrey, Guildford, Surrey GU2 7XH, United Kingdom \\ ${ }^{4}$ Centro de Física Computacional, Department of Physics, University of Coimbra, PT-3004-516 Coimbra, Portugal
}

(Received 26 July 2012; revised manuscript received 26 October 2012; published 3 December 2012)

\begin{abstract}
We present calculations of the energy per particle of pure neutron and symmetric nuclear matter with simplified Argonne nucleon-nucleon potentials for different many-body theories. We compare critically the BruecknerHartree-Fock results to other formalisms, such as the Brueckner-Bethe-Goldstone expansion up to third order, self-consistent Green's functions, auxiliary field diffusion Monte Carlo, and Fermi hypernetted chain. We evaluate the importance of spin-orbit and tensor correlations in the equation of state and find these to be important in a wide range of densities.
\end{abstract}

DOI: 10.1103/PhysRevC.86.064001

PACS number(s): 13.75.Cs, 21.30.Fe, 24.10.Cn, 21.65.Mn

\section{INTRODUCTION}

The properties of homogeneous nuclear and neutron matter at high density play a crucial role in the determination of the structure of neutron-star interiors [1]. Terrestrial nuclei provide little input to constrain the equation of state (EOS) under the extreme conditions of density and isospin asymmetry within neutron stars. A potentially safe way to obtain the EOS thus relies on microscopic many-body calculations based on realistic nucleon-nucleon $(N N)$ interactions. Over the years, several many-body approaches have been developed to describe neutron-star (and nuclear) interiors. The different approaches might have very different physical foundations, but their final result is generally the same: a prediction for the density dependence of the energy per particle in neutron or symmetric nuclear matter. Here we want to progress in our understanding of the EOS by comparing quantitatively the results provided by different many-body approaches. Similar benchmark calculations have taken place within the few-body community and have provided vital insight into the approximation schemes at play [2].

The full operatorial structure of current high-quality $N N$ potentials is presently too sophisticated for some state-of-theart many-body schemes. Simplified versions of these potentials are therefore useful for benchmarking purposes. In particular, we use a family of simpler versions of the widely used Argonne $V_{18}$ potential [3]. The $V_{8}^{\prime}, V_{6}^{\prime}$, and $V_{4}^{\prime}$ potentials [4] are built by removing operatorial components of the interaction, while the remaining terms are readjusted (as indicated by the prime) to preserve as many lowest-order phase shifts and deuteron properties as possible. These potentials have been used in calculations of both finite nuclei [4] and infinite matter (see, e.g., Refs. [5] and [6]). A natural question then arises: How well can these truncated potentials replace the original $V_{18}$ ?

To clarify this and other issues, we first analyze in detail the properties of the family of Argonne potentials by inspecting their phase shifts in different partial waves. We also examine the deuteron properties as predicted by these interactions. We then study the EOS within the Brueckner-Hartree-Fock (BHF) many-body approach [7], which can handle straightforwardly all the variants of the Argonne potential. The BHF results are particularly insightful, because the total energy can be directly connected to the partial wave expansion and therefore to the microscopic properties of the in-medium $N N$ interaction. We also compare, when possible, the BHF results with other many-body approaches [8,9], namely the Brueckner-BetheGoldstone (BBG) approach up to third order in the hole-line expansion [10], the self-consistent Green's function (SCGF) method [11-13], the auxiliary field diffusion Monte Carlo (AFDMC) [6], the Green's function Monte Carlo (GFMC) [5], and the Fermi hypernetted chain (FHNC) [14-16]. The comparisons should be helpful in quantifying theoretical uncertainties with respect to the EOS. As we shall see, the symmetric nuclear matter predictions are particularly susceptible to the missing spin-orbit components in simplified potentials.

This work complements and extends previous investigations of some of the authors [17,18]. In fact, Ref. [18] contains BHF results for $V_{18}, V_{8}^{\prime}$, and $V_{6}^{\prime}$ potentials. We note, however, that all BHF results for these potentials shown in the present work have been recalculated and that the results corresponding to $V_{4}^{\prime}$ and $\tilde{V}_{6}$ have never been reported before. Regarding the BBG results, Ref. [17] reports calculations for $V_{18}$ and $V_{8}^{\prime}$ for both symmetric and neutron matter which have been carefully revised in the present paper. All the other BBG results are new and presented for the first time. The SCGF results presented here correspond to the zero-temperature extrapolation of results presented in previous publications [11]. The extrapolation procedure has not yet been discussed. As such, the results should be taken as new, because this is the first time they are published. The comparisons with the FHNC results are performed with the most recent FHNC calculations for symmetric and neutron matter [16,19]. Finally, the AFDMC results were provided by Gandolfi [20]. Notice, however, that the AFDMC results for $V_{6}^{\prime}$ and $V_{8}^{\prime}$ are those shown in Ref. [16]. Our basic conclusions are related to the spin-orbit and tensor components of the $N N$ interaction and $N N$ in-medium correlations. Let us stress, in addition, that we do not attempt to obtain a "realistic" description of 
the neutron or symmetric nuclear-matter EOS. Our goal is solely to compare constructively the results obtained with different two-body potentials and many-body methods. A detailed calculation of the nuclear EOS would need to consider additional effects, particularly three-body forces [21] and relativistic corrections, which are beyond the scope of the present work.

The paper is organized as follows. In Secs. II and III we show the phase shifts and the deuteron properties predicted by the different Argonne potentials. Section IV is devoted to the BHF results of nuclear matter, both symmetric matter (SM) and neutron matter (NM), whereas in Sec. V we compare the results obtained with the different many-body approaches. Finally, our conclusions are presented in Sec. VI.

\section{PHASE SHIFTS}

The strong interaction part of the Argonne $V_{18}$ potential can be expressed as a sum of 18 operators,

$$
V_{i j}=\sum_{p=1,18} v_{p}\left(r_{i j}\right) O_{i j}^{p} .
$$

The first 14 operators are associated to the spin, isospin, tensor, spin-orbit, and quadratic spin-orbit components of the nuclear force:

$$
\begin{aligned}
O_{i j}^{p=1, \ldots, 14} & \\
= & 1, \boldsymbol{\tau}_{i} \cdot \boldsymbol{\tau}_{j}, \boldsymbol{\sigma}_{i} \cdot \boldsymbol{\sigma}_{j},\left(\boldsymbol{\sigma}_{i} \cdot \boldsymbol{\sigma}_{j}\right)\left(\boldsymbol{\tau}_{i} \cdot \boldsymbol{\tau}_{j}\right), \\
& S_{i j}, S_{i j}\left(\boldsymbol{\tau}_{i} \cdot \boldsymbol{\tau}_{j}\right), \\
& \boldsymbol{L} \cdot \boldsymbol{S}, \boldsymbol{L} \cdot \boldsymbol{S}\left(\boldsymbol{\tau}_{i} \cdot \boldsymbol{\tau}_{j}\right), \\
& L^{2}, L^{2}\left(\boldsymbol{\tau}_{i} \cdot \boldsymbol{\tau}_{j}\right), L^{2}\left(\boldsymbol{\sigma}_{i} \cdot \boldsymbol{\sigma}_{j}\right), L^{2}\left(\boldsymbol{\sigma}_{i} \cdot \boldsymbol{\sigma}_{j}\right)\left(\boldsymbol{\tau}_{i} \cdot \boldsymbol{\tau}_{j}\right), \\
& (\boldsymbol{L} \cdot \boldsymbol{S})^{2},(\boldsymbol{L} \cdot \boldsymbol{S})^{2}\left(\boldsymbol{\tau}_{i} \cdot \boldsymbol{\tau}_{j}\right) .
\end{aligned}
$$

The four additional operators,

$$
O_{i j}^{p=15, \ldots, 18}=T_{i j}, T_{i j}\left(\sigma_{i} \cdot \sigma_{j}\right), T_{i j} S_{i j},\left(\tau_{z i}+\tau_{z j}\right),
$$

where $T_{i j}=3 \tau_{z i} \tau_{z j}-\boldsymbol{\tau}_{i} \cdot \boldsymbol{\tau}_{j}$ is the isotensor operator, break charge independence. The radial functions that multiply each operator are adjusted by fitting experimental data on two-body scattering phase shifts as well as deuteron properties.

A family of simplified Argonne $N N$ interactions has been devised to quantify the evolution of nuclear spectra with increasingly sophisticated $N N$ interactions [4]. A given simplified version, $V_{n}^{\prime}$, is constructed by (i) eliminating the operatorial structure with $p>n$ and (ii) refitting the remaining radial functions to reproduce as many properties of the original interaction as possible. Thus, $V_{8}^{\prime}$ includes all operators up to the spin-orbit term, but misses the components proportional to $L^{2},(\boldsymbol{L} \cdot \boldsymbol{S})^{2}, T_{i j}$, and $\tau_{z i}+\tau_{z j}$. Similarly, $V_{6}^{\prime}$ is a $N N$ force without spin-orbit (or further, $p>6$ ) couplings. Finally, $V_{4}^{\prime}$ does not even have a tensor coupling, but has been refitted to reproduce the binding energy of the deuteron.

Realistic (or microscopic) $N N$ interactions should fulfill a minimum set of requirements. In particular, realistic potentials are built to reproduce the Nijmegen database [22] (which contains a full set of $N N$ elastic scattering phase shifts up to energies of about $350 \mathrm{MeV}$ ) with an accuracy of $\chi^{2} / N_{\text {data }} \sim 1$. Only potentials that fulfill this condition should be used as input to the so-called $a b$ initio many-body schemes, which aim at providing a first-principles description of the EOS of $\mathrm{NM}$ and SM. While Argonne $V_{18}$ is, by all means, a realistic interaction, the Argonne family of simpler versions will necessarily violate this condition and thus they will become, in some sense, increasingly "less realistic." In particular, one expects the reproduction of the phase shifts of high partial waves to be deteriorated as the operatorial structure of the interaction is simplified. One of our aims is to explore to which extent this deterioration has an impact on the EOS. This is particularly important in view of the fact that some approaches, at present, are limited to simplified forms of the Argonne family of potentials. In particular, the last generation of FHNC results has been computed with interactions up to $V_{8}^{\prime}$ for both SM and NM. For ADFMC, the EOS of NM (SM) is only available with $V_{8}^{\prime}\left(V_{6}^{\prime}\right)$. Diagrammatic approaches, such as BHF, BBG, or SCGF, have fewer limitations with respect to the structure of the original $N N$ interactions. We therefore provide results for Argonne $V_{18}$ for these approaches. Within a given many-body approximation, calculations with $V_{18}, V_{8}^{\prime}, V_{6}^{\prime}$, and $V_{4}^{\prime}$ should provide an indication of the importance of the missing operatorial components for the in-medium properties.

Figure 1 shows the phase shifts of the lowest partial waves given by the different Argonne potentials [4]. $V_{18}, V_{8}^{\prime}$, and $V_{6}^{\prime}$ agree, by construction, in the ${ }^{1} S_{0},{ }^{3} S_{1},{ }^{1} P_{1}$, and ${ }^{3} D_{1}$ partial waves. The oversimplistic $V_{4}^{\prime}$ potential, however, does not have a tensor coupling, thus yielding zero mixing angles $\varepsilon_{1}$ and $\varepsilon_{2}$. The ${ }^{3} D_{1}$ phase shift is also badly reproduced for this potential, with an opposite sign relative to the other potentials. This is a direct consequence of the readjustment of the potential to bind the deuteron with the $S$ channel only [4]. In the ${ }^{3} D_{1}$ wave, the readjustment is such that it changes the nature of the interaction from repulsive (negative phase-shift) to attractive (positive phase-shift).

For $L=1$, further discrepancies between phase shifts appear. All potentials reproduce the ${ }^{1} P_{1}$ phase-shift correctly, as the spin-orbit or tensor components are not active. Substantial differences, however, show up already in the ${ }^{3} P_{0,1,2}$ waves. In these channels, the $V_{6}^{\prime}$ and $V_{4}^{\prime}$ potentials deviate from the experimentally fitted $V_{18}$ results. In particular, the important ${ }^{3} P_{2}$ wave is grossly misrepresented with the $V_{6}^{\prime}$ potential. In fact, this potential provides no correction in the $S=1, T=1$ partial waves for the missing spin-orbit components and can hardly be considered realistic for this reason.

Similarly large discrepancies are observed for the phase shifts of the ${ }^{1} D_{2}$ and ${ }^{3} D_{2}$ partial waves. In the $S=0$ channel, $V_{6}^{\prime}$ and $V_{4}^{\prime}$ are identical and provide a too large phase shift compared to $V_{18}$ and $V_{8}^{\prime}$ (which lie on top of each other in the plot). In the $S=1$ channel, visible differences show up also between $V_{8}^{\prime}$ and $V_{18}$. For the $L=3$ phase shifts, we have chosen to show the ${ }^{3} F_{2}$ channel, where, again, substantial and visible differences appear between $V_{4}^{\prime}, V_{6}^{\prime}, V_{8}^{\prime}$, and $V_{18}$.

The lowest right panels of Fig. 1 show the mixing parameters $\varepsilon_{1}$ and $\varepsilon_{2}$ of the ${ }^{3} S_{1}-{ }^{3} D_{1}$ and ${ }^{3} P_{2}-{ }^{3} F_{2}$ partial waves, respectively. On the one hand, the $V_{8}^{\prime}$ interaction reproduces very well the behavior of both parameters as given by the full $V_{18}$, in spite of the slightly different ${ }^{3} F_{2}$ phase shift. On the other hand, $V_{6}^{\prime}$ is only able to account for the low-energy behavior $\left(E_{\text {lab }} \leqslant 50 \mathrm{MeV}\right)$ of $\varepsilon_{1}$ and $\varepsilon_{2}$. $V_{4}^{\prime}$ lacks a tensor 

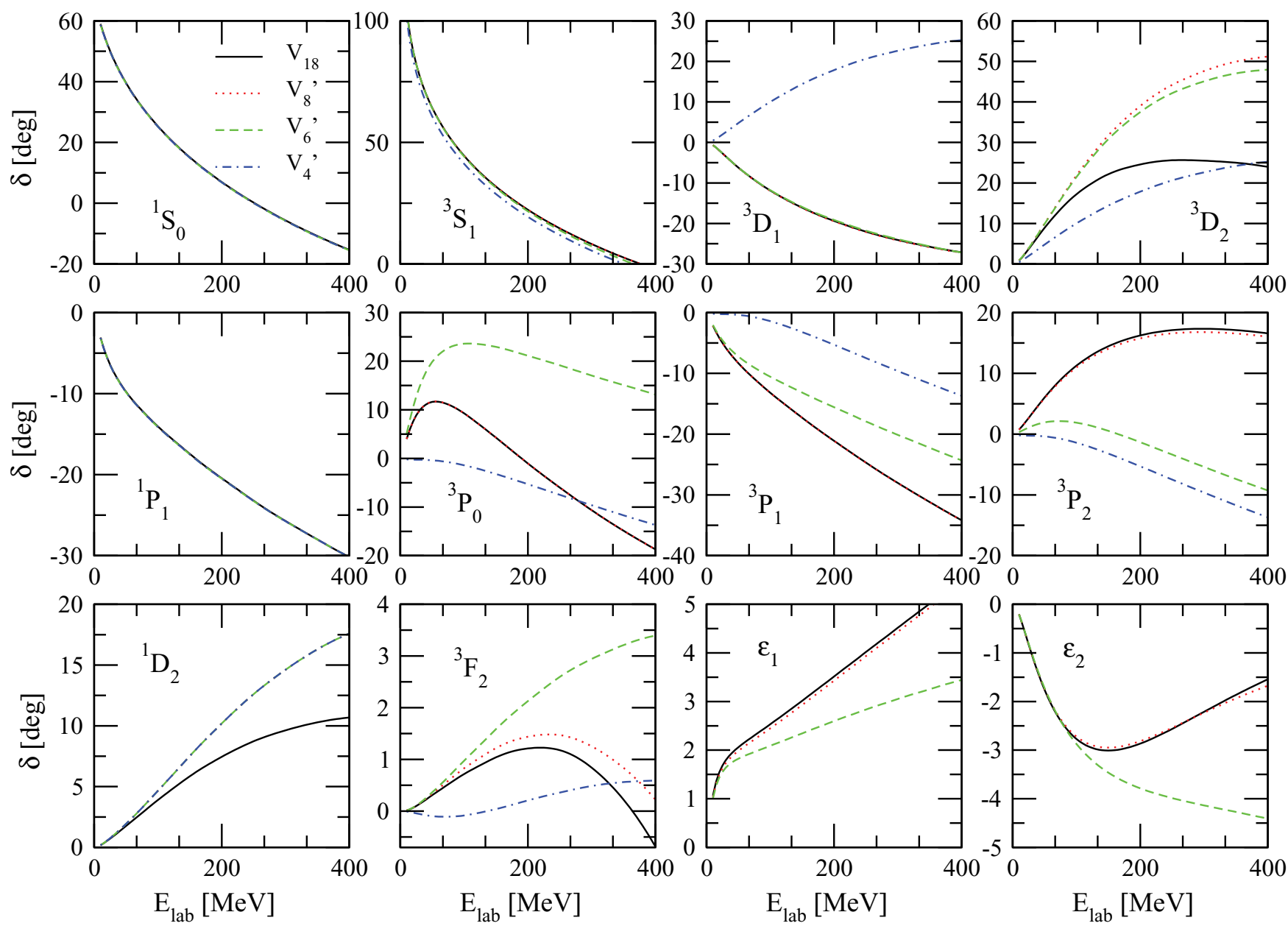

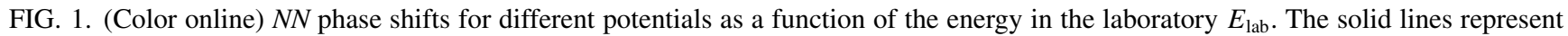
the reference Argonne $V_{18}$ results, whereas dotted, dashed, and dashed-dotted lines correspond to $V_{8}^{\prime}, V_{6}^{\prime}$, and $V_{4}^{\prime}$, respectively.

component and therefore the mixing parameters associated to it are zero.

Let us note, for further reference, that the BHF calculations are performed with partial waves up to $J=8$. The SCGF results presented here have been obtained for up to $J=4$ in the $T$-matrix and up to $J=8$ in the Hartree-Fock self-energy. For the BBG three-hole calculations, all contributions have $J \leqslant 5$ and the convergence has been carefully tested. The differences in the $J>1$ partial waves that we have just highlighted will therefore have an impact on the EOS. As a matter of fact, even phase-shift-equivalent potentials might predict different EOS owing to their different (and physically unconstrained) offshell structures. Naturally, one would expect these differences to be small at low densities, where the physics is mainly dominated by $L=0$ components. As the density increases, however, the differences in the higher partial waves start to show up. In nonperturbative diagrammatic calculations, the effects of high partial waves can be fed back to low momenta through the self-consistency procedure. Likewise, relatively small differences in the phase shifts can have a substantial impact on the predictions of the EOS at densities even close to saturation.

\section{DEUTERON PROPERTIES}

Even though the deuteron only explores the $N N$ potential in the ${ }^{3} S_{1}-{ }^{3} D_{1}$ partial waves, the analysis of the contributions of the different waves and operatorial components of the interaction provides a useful insight into the structure of the interaction [23]. We summarize the information related to the deuteron in Table I. The first column gives the $D$-state probability computed with different $N N$ interactions. Although $P_{D}$ is not an observable, it provides an indication of the relative importance of the tensor coupling of the potential. By construction, the $D$-state probability of $V_{8}^{\prime}$ is the same as that of $V_{18}, P_{D}=5.78 \%$. For $V_{6}^{\prime}$ the probability decreases by less than $10 \%$. As expected, $P_{D}=0$ for $V_{4}^{\prime}$, whose deuteron is a pure $S$-wave state. We also include the results of an additional potential, $\tilde{V}_{6}$, obtained by removing, without any readjustment, the spin-orbit components from $V_{8}^{\prime}$. For $\tilde{V}_{6}, P_{D}$ is reduced by almost $20 \%$, indicating the importance of the spin-orbit components [ $p=7,8$ in Eq. (2)] for the ${ }^{3} D_{1}$ wave and thus the ground state of the deuteron. Similar statements hold for the quadrupole moment of the deuteron, which is an observable. As such, $Q_{d}$ is reproduced, by construction, with the original $V_{18}$ force and the refitted $V_{8}^{\prime}$ and $V_{6}^{\prime}$ forces. The 
TABLE I. Deuteron $D$-state probability $P_{D}$, quadrupole moment $Q_{d}$ (in $\mathrm{fm}^{2}$ ), total binding energy, kinetic and potential energy, and their decomposition in partial waves, for different potentials. All energies are given in MeV.

\begin{tabular}{lcccccccccc}
\hline \hline Force & $P_{D}(\%)$ & $Q_{d}$ & $E$ & $T$ & $V$ & $T_{S}$ & $T_{D}$ & $V_{S}$ & $V_{D}$ & $2 V_{S D}$ \\
\hline$V_{18}$ & 5.78 & 0.27 & -2.24 & 19.86 & -22.10 & 11.30 & 8.56 & -3.95 & 0.77 & -18.91 \\
$V_{8}^{\prime}$ & 5.78 & 0.27 & -2.24 & 19.86 & -22.10 & 11.30 & 8.56 & -3.95 & 0.77 & -18.91 \\
$V_{6}^{\prime}$ & 5.33 & 0.27 & -2.24 & 18.70 & -20.94 & 11.38 & 7.32 & -4.68 & 1.38 & -17.64 \\
$V_{4}^{\prime}$ & 0.00 & 0.00 & -2.24 & 11.41 & -13.65 & 11.41 & 0.0 & -13.65 & 0.0 & 0.0 \\
$\tilde{V}_{6}$ & 4.64 & 0.30 & -1.46 & 14.96 & -16.42 & 9.10 & 5.86 & -3.43 & 1.14 & -14.14 \\
\hline \hline
\end{tabular}

lack of tensor components in $V_{4}^{\prime}$, however, implies a zero value of $Q_{d}$. The non-refitted interaction $\tilde{V}_{6}$ yields a value which is about $10 \%$ larger than the experimental one.

In columns $3-5$, we report the binding energy of the deuteron and its decomposition into kinetic and potential terms. All the refitted potentials reproduce by construction the total binding energy, $E=-2.24 \mathrm{MeV}$. Note that this is the binding energy obtained only with the strong interaction components of the potential, that is, when the small electromagnetic terms are omitted. These repulsive electromagnetic terms shift the binding energy to the true experimental value of $E=-2.22 \mathrm{MeV}$ [4]. It is also relevant to note that the charge-dependent terms of $V_{18}(p=15, \ldots, 18)$, described in terms of an isotensor operator, have no contribution in the isosinglet deuteron state.

It is well known that the deuteron binding energy results from a cancellation between a large positive kinetic and a large negative potential energy. For $V_{18}$ these amount to $T=19.86 \mathrm{MeV}$ and $V=-22.10 \mathrm{MeV}$, respectively. By construction, $V_{8}^{\prime}$ reproduces the same values as $V_{18}$. For $V_{6}^{\prime}$ there is a small variation in $T$ and $V$ because the ${ }^{3} S_{1}$ and ${ }^{3} D_{1}$ partial waves are not exactly identical to those of the $V_{18}$ and $V_{8}^{\prime}$ potentials. In particular, the kinetic energy decreases by about $1 \mathrm{MeV}$. $V_{4}^{\prime}$ is also able to reproduce the total binding of the deuteron, but with much smaller kinetic (and therefore less negative potential) energies. In contrast, the potential $\tilde{V}_{6}$ loses binding energy and also produces very noticeable differences for the kinetic and potential energies. In other words, a straightforward elimination of the spin-orbit components, without further readjustments, has large effects for the binding energy.

It is also illustrative to separate the contributions of the ${ }^{3} S_{1}$ and ${ }^{3} D_{1}$ states to the total kinetic and potential energies. Assuming that the deuteron is a properly normalized combination of the ${ }^{3} S_{1}$ and ${ }^{3} D_{1}$ partial waves, we define the contributions of the $S$ and $D$ states to the kinetic energy, $T_{S}=\left\langle{ }^{3} S_{1} \mid T{ }^{3} S_{1}\right\rangle$ and $T_{D}=\left\langle{ }^{3} D_{1}|T|^{3} D_{1}\right\rangle$, and to the potential energy, $V_{S}=\left\langle{ }^{3} S_{1}|V|{ }^{3} S_{1}\right\rangle$ and $V_{D}=\left\langle{ }^{3} D_{1}|V|{ }^{3} D_{1}\right\rangle$. The latter also receives a contribution from the ${ }^{3} S_{1}{ }^{3} D_{1}$ mixing, $V_{S D}=$ $\left.{ }^{3} S_{1}|V|{ }^{3} D_{1}\right\rangle$. These contributions are listed, for the different potentials, in columns 6 to 10 of Table I.

For $V_{18}, V_{8}^{\prime}$, and $V_{6}^{\prime}$, the largest contribution to the potential energy actually comes from the mixing term, $V_{S D}$. This accounts for more than $85 \%$ of the final value of the potential energy. As mentioned above, for $V_{4}^{\prime}$ the deuteron is a pure $S$-wave state, and therefore $T_{D}, V_{D}$, and $V_{S D}$ vanish and the binding energy is obtained by accumulating a lot of attraction in $V_{S}$. In spite of the fact that the spin-orbit components of $V_{18}$ and $V_{8}^{\prime}$ act explicitly only in the ${ }^{3} D_{1}$ partial wave, when these are eliminated without readjustments in $\tilde{V}_{6}$, all the contributions to the binding energy (and not only $V_{D}$ ) are altered. This is attributable to the fact that, owing to the tensor coupling, the deuteron is obtained nonperturbatively from a combination of $S, D$, and mixing matrix elements. One can therefore say that when the spin-orbit component is not taken into account, a large change is induced in the wave function of the deuteron. In a wider picture, these results illustrate how the elimination of operatorial components can have a relatively large impact in the binding energy of nuclear systems. The changes and differences induced by such elimination become more apparent when different channels are analyzed separately. While the refitting procedure in the $V_{n}^{\prime}$ family of potentials seems to cure most deficiencies in the case of the deuteron, no such analogous procedure has been implemented in infinite matter. Consequently, one expects that even refitted potentials have a significant influence on the EOS of the infinite system.

Alternatively, one can obtain a quantitative estimation of the different components of the potential by examining their expectation values in the ground-state wave function of the deuteron. We have grouped the 18 components into four different sets: the first four operators $(p=1, \ldots, 4)$, the tensor components $S_{i j}(p=5,6)$, the spin-orbit components $\boldsymbol{L} \cdot \boldsymbol{S}$ and $(\boldsymbol{L} \cdot \boldsymbol{S})^{2}(p=7,8$ and $p=13,14)$, and the quadratic orbital angular momentum components $L^{2}(p=9, \ldots, 12)$. The group of charge-dependent terms, $p=15, \ldots, 18$, does not contribute to the deuteron, as explained above. For $V_{18}$, the results of this decomposition are presented in the first row of Table II. As expected, the largest contribution corresponds to the tensor component. All contributions are attractive, except that proportional to $L^{2}$, which is slightly repulsive.

TABLE II. Contribution of different components of the potential to the binding energy of the deuteron. All energies are given in $\mathrm{MeV}$. See text for details.

\begin{tabular}{lrrrr}
\hline \hline Force & Central & Tensor & Spin orbit & \multicolumn{1}{c}{$L^{2}$} \\
\hline$V_{18}$ & -4.45 & -16.62 & -3.75 & 2.72 \\
$V_{8}^{\prime}$ & -4.45 & -16.62 & -1.02 & 0.00 \\
$V_{6}^{\prime}$ & -5.25 & -15.69 & 0.00 & 0.00 \\
$V_{4}^{\prime}$ & -13.65 & 0.00 & 0.00 & 0.00 \\
$\tilde{V}_{6}$ & -3.84 & -12.58 & 0.00 & 0.00 \\
\hline \hline
\end{tabular}


The spin-orbit contribution is non-negligible and amounts to $17 \%$ of the total potential energy.

The second row of Table II shows the results for $V_{8}^{\prime}$. The total potential energy, that is, the sum of all the components, is the same as for $V_{18}$. However, because the contribution of the first six operators yield the same result as $V_{18}$ and the quadratic orbital angular momentum contribution is zero, the contribution of the spin-orbit terms is reduced to $-1.02 \mathrm{MeV}$. In other words, the absence of the repulsive $L^{2}$ component is compensated by a smaller attractive contribution of the spin-orbit components, which decreases to $4.6 \%$ of the total potential energy.

For $V_{6}^{\prime}$ only the first six operators contribute (row 3 in Table II). These components are about $1 \mathrm{MeV}$ different from those in $V_{18}$ and produce a slightly smaller total potential energy compared to the full results. As we have discussed previously, for $\tilde{V}_{6}$ there is an important loss of binding energy. It is interesting to notice the difference between the spin-orbit contribution provided by $V_{8}^{\prime}(-1.02 \mathrm{MeV})$ and the expectation value of the $\boldsymbol{L} \cdot \boldsymbol{S}$ component of $V_{8}^{\prime}$ in the ground-state wave function provided by $\tilde{V}_{6}$, which amounts to $-0.58 \mathrm{MeV}$. Obviously, this difference is attributable to the different wave functions generated by the two potentials. The wave function associated with $\tilde{V}_{6}$ does not contain the spin-orbit correlations that are generated by solving the Schrödinger equation with $V_{8}^{\prime}$. Therefore, the evaluation of the expectation value of the $\boldsymbol{L} \cdot \boldsymbol{S}$ component of $V_{8}^{\prime}$ in the wave function provided by $\tilde{V}_{6}$ gives a poor estimate of the spin-orbit contribution of $V_{8}^{\prime}$. This suggests that it is necessary to incorporate the spin-orbit correlations in the wave function to recover the full contribution. However, the expectation value of the $\boldsymbol{L} \cdot \boldsymbol{S}$ component of $V_{8}^{\prime}$ in the ground-state wave function provided by $V_{6}^{\prime}$ amounts to $-0.77 \mathrm{MeV}$, which lies in between the above discussed results. One can interpret that the refitting process that constrains $V_{6}^{\prime}$ to reproduce phase shifts and the deuteron binding energy translates in a wave function closer to the ground-state provided by $V_{8}^{\prime}$. Consequently, the expectation value of the $\boldsymbol{L} \cdot \boldsymbol{S}$ component is closer to the exact value than the results provided by $\tilde{V}_{6}$. Notice, however, that it would not make sense to add this $\boldsymbol{L} \cdot \boldsymbol{S}$ contribution to the binding energy because the potential $V_{6}^{\prime}$ (with no spin-orbit component) already reproduces by construction the binding energy of the deuteron. The deuteron results illustrate effectively the non-negligible significance of spin-orbit effects both on the binding energy and the wave function.

\section{NUCLEAR EOS IN THE BHF APPROACH}

We now proceed to compare the results for the EOS of SM and NM obtained within the BHF approach for the family of simplified Argonne potentials described above. The BHF approach represents the lowest order within the BBG expansion $[7,10]$. In this formalism, the ground-state energy of infinite matter is computed from a diagrammatic expansion, which is regrouped according to the number of independent hole lines. Within the BHF approach, the energy is given by the sum of only two-hole-line diagrams, including the effect of two-body correlations through the in-medium two-body scattering $G$-matrix. This takes into account the effect of the Pauli principle on the scattered particles and the in-medium potential felt by each nucleon.

As shown in Refs. [10], the contribution to the energy from three-hole-line diagrams (which account for the effect of threebody correlations) is minimized when the so-called continuous prescription [24] for the single-particle potential is adopted. This is a strong indication of the convergence of the hole-line expansion and we have adopted this prescription in our BHF calculations. It has to be stressed that three or higher holeline contributions can be negligibly small only because of the inclusion of the so-called U-insertion diagrams [7,10], which is a distinct feature of the BBG method, not present in other schemes. We would also like to mention that the $G$-matrix has been calculated both in $r$ and $k$ space with two independent numerical codes and an excellent agreement has been found at all densities. We also remind the reader that no three-body forces are included in our calculations.

In general, owing to their different off-shell behavior, phaseshift equivalent potentials might lead to different saturation curves of nuclear matter. Within BHF, the saturation points of phase-shift equivalent interactions lie on the so-called "Coester band," which provides an empirical correlation between the saturation energy and density [25]. Quite obviously, potentials with different phase shifts will also predict different saturation properties for infinite matter. We see in the following that a "Coester-like" correlation holds, however, for the different members of the simplified Argonne family of interactions.

In Fig. 2, we compare the total energies of SM (top panel) and NM (bottom panel) within the BHF approach for the different Argonne $N N$ forces [7]. Several striking features arise from these comparisons. To begin with, one immediately notices the relatively large differences between the $V_{18}$ (circles) and the $V_{8}^{\prime}$ (squares) results in both cases. This is rather surprising, especially in view of the close agreements between phase shift and deuteron results presented earlier. In both SM and NM, $V_{8}^{\prime}$ predicts a much more attractive EOS compared to $V_{18}$. In contrast, the $V_{6}^{\prime}$ results (diamonds) are fairly close to the $V_{18}$ calculations, in spite of their relatively different phase shifts and deuteron predictions. The $V_{4}^{\prime}$ results are relatively reasonable for $\mathrm{NM}$, where the ${ }^{3} S D_{1}$ channel is absent, while they are clearly unrealistic for SM. As a matter of fact, SM does not even saturate before $\rho=0.5 \mathrm{fm}^{-3}$ for this potential. For a more detailed insight, we refer the reader to Table III, where the energies of SM and NM at different densities are listed for the Argonne family of potentials.

Another relevant question associated to the spin-orbit coupling arises when comparing the results predicted by the $V_{6}^{\prime}$ and $\tilde{V}_{6}$ potentials. $V_{6}^{\prime}$ has been constructed specifically by modifying only the central potential in the $T=0, S=1$ states [4]. As a consequence, both potentials are identical in the $T=1$ partial waves and therefore they predict the same EOS for pure NM (see bottom panel of Fig. 2). The effect of correcting the central $T=0, S=1$ term is only seen in $\mathrm{SM}$ and corresponds to a rather density-independent gain of binding of a few $\mathrm{MeV}$ with respect to the $\tilde{V}_{6}$ results (see top panel of Fig. 2).

The BHF approach is almost unique within all the manybody approaches, in the sense that the total energy of the 

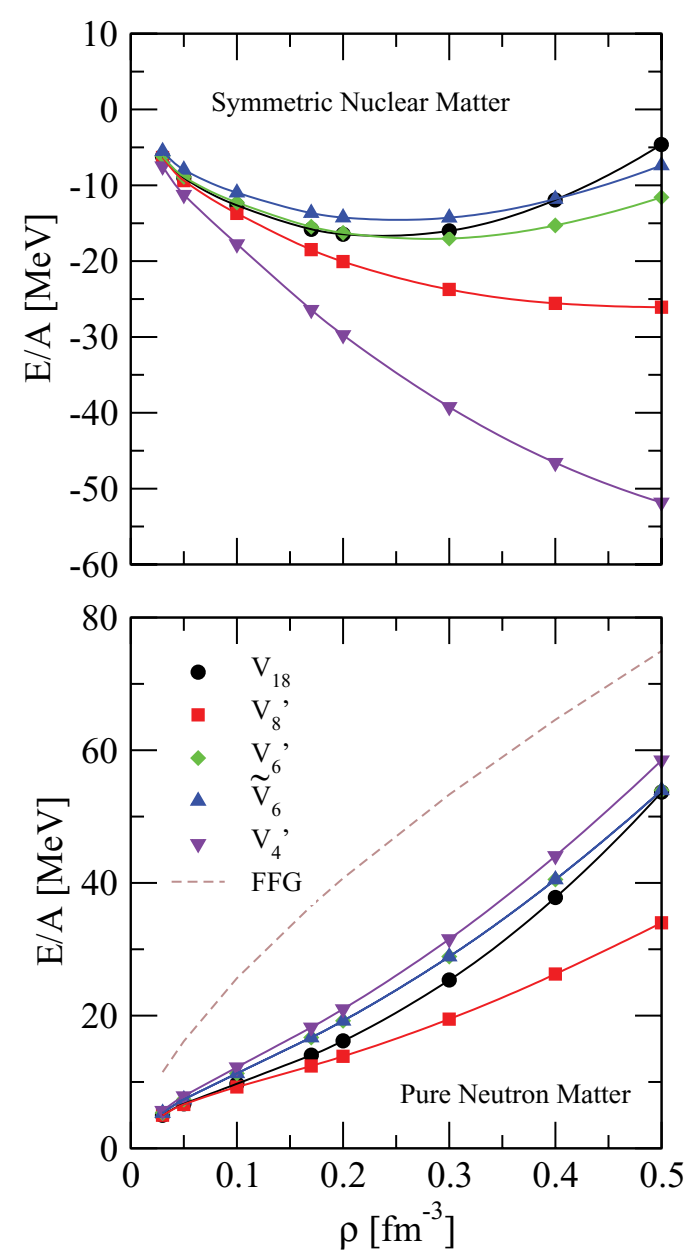

FIG. 2. (Color online) Energy per particle for nuclear (top panel) and neutron (bottom panel) matter as a function of density calculated in the BHF approach with the different Argonne potentials.

system can be linked to the partial wave expansion of the in-medium $N N$ interaction. This provides an interesting insight into the impact of different partial waves on the EOS and has motivated us to use BHF as a reference calculation. An analysis of the different partial wave contributions to the total energy at $\rho=0.3 \mathrm{fm}^{-3}$ is listed in Table IV for SM and NM. In SM, the largest difference between the $V_{18}$ and $V_{8}^{\prime}$ results arises from the ${ }^{3} S D_{1}$ wave $(4.8 \mathrm{MeV})$, even though the phase shifts in these coupled channels are practically identical. We have verified that this effect is actually attributable to the different single-particle potentials. The differences in higherorder partial waves thus influence, via the single-particle states, the lowest partial waves. Other substantial contributions to the difference between $V_{18}$ and $V_{8}^{\prime}$ stem from the ${ }^{1} S_{0}(1.3 \mathrm{MeV})$, the ${ }^{1} D_{2}(0.8 \mathrm{MeV})$, and the ${ }^{3} D_{2}(0.9 \mathrm{MeV})$ states.

Somewhat surprisingly, the total energy of $V_{6}^{\prime}$ is actually closer to that of $V_{18}$, even though the individual partial wave contributions are rather different. For instance, the ${ }^{3} P F_{2}$ energy difference is very large $(-12.3 \mathrm{MeV})$, in agreement with the unrealistic phase shifts of $V_{6}^{\prime}$ in this channel. This large discrepancy, however, is canceled by opposite (and also relatively large) differences in the ${ }^{3} P_{0}$ and ${ }^{3} P_{1}$ channel. We specify the sum of all ${ }^{3} P$ states in the fifth row of Table IV.
This shows that, in the case of $V_{6}^{\prime}$, all ${ }^{3} P$ waves amount to a difference of only $-3.3 \mathrm{MeV}$ in the total energy. Together with the opposite-sign differences in the ${ }^{3} S D_{1}(3.5 \mathrm{MeV})$ and ${ }^{3} D_{2}$ channels $(0.8 \mathrm{MeV})$, the total energy eventually becomes close to the $V_{18}$ value.

As we have just discussed, the binding energies (and saturation curves) of SM obtained with the $V_{8}^{\prime}$ and the $V_{6}^{\prime}$ potentials are rather different. These large differences are associated with the phase-shift nonequivalence of the two interactions. Note, in particular, that the largest differences between the two sets of results manifest themselves in the channels where the two potentials are actually not equivalent (i.e., where $V_{6}^{\prime}$ has been modified with respect to $V_{8}^{\prime}$ ). The different mixing of the ${ }^{3} S D_{1}$ channels induces a difference of $1.3 \mathrm{MeV}$. Once again, even though the ${ }^{3} P$ individual contributions are rather different, the overall sum leads to a relatively small difference of $4.1 \mathrm{MeV}$. This is the largest contribution to the total binding energy difference of $6.9 \mathrm{MeV}$. Because the energies associated with $V_{8}^{\prime}$ are more attractive, a higher saturation density is found in comparison with $V_{18}$ and $V_{6}^{\prime}$. We conclude that an intricate series of compensations leads to closer agreement between $V_{6}^{\prime}$ and $V_{18}$ than between $V_{8}^{\prime}$ and $V_{18}$. This is clearly misleading in view of the insufficient phase shift reproduction of $V_{6}^{\prime}$ compared to $V_{8}^{\prime}$.

A similar issue is observed in the partial wave decomposition of the $V_{4}^{\prime}$ results. The $T=1$ partial waves disagree substantially on a one-by-one basis (in some cases by more than $10 \mathrm{MeV}$ ), but the overall sum of the ${ }^{3} P$ waves differs from the $V_{18}$ results by a smaller number, $-6.8 \mathrm{MeV}$. The most extreme difference is obtained, as expected, in the ${ }^{3} S D_{1}$ channel, which is about $20 \mathrm{MeV}$ more attractive for $V_{4}^{\prime}$ than for the rest of the potentials. Ultimately, it is this extreme additional binding, caused by the lack of partial-wave coupling and the extreme readjustment of the ${ }^{3} D_{1}$ channel, that drives the nonsaturating behavior of $V_{4}^{\prime}$.

The partial-wave differences in NM (see right columns in Table IV) also carry interesting information. On a channelby-channel basis, the comparison between $V_{18}$ and $V_{8}^{\prime}$ results is more favorable than that between $V_{18}$ and $V_{6}^{\prime}$. Yet, once again, the discrepancies are reduced in the overall sum, so that the $V_{6}^{\prime}$ final results are closer to $V_{18}$. At $\rho=0.3 \mathrm{fm}^{-3}$, $\mathrm{NM}$ is about $10 \mathrm{MeV}$ more bound with the $V_{8}^{\prime}$ than with $V_{6}^{\prime}$. This difference is entirely attributable to the spin-orbit component. In particular, the phase-shift nonequivalence in the ${ }^{3} P F_{2}$ channel is evidenced by a difference of more than $20 \mathrm{MeV}$. Similarly, the $V_{4}^{\prime}$ results for NM are only slightly more repulsive than the others. The partial-wave decomposition suggests that this is caused by to a reshuffling of partial wave contributions which would, separately, deviate substantially from more realistic results.

Finally, we would like to comment on the specific effect of the spin-orbit components in a neutron-rich medium. First, notice that in NM the differences between $V_{6}^{\prime}=\tilde{V}_{6}$ and $V_{8}^{\prime}$ are only attributable to the suppression of the spin-orbit components in $V_{8}^{\prime}$, as no other readjustments are applied. Clearly, the elimination (rather than the readjustment) procedure for spin-orbit components in the interaction does not have a small effect in the EOS of NM, as evidenced by the large differences between the $V_{6}^{\prime}$ and $V_{8}^{\prime}$ results in Fig. 2. 
TABLE III. Energies per particle (in MeV) for SM and NM at different densities and for different interactions calculated within the BHF approximation.

\begin{tabular}{|c|c|c|c|c|c|c|c|c|}
\hline \multirow[t]{2}{*}{$\rho\left(\mathrm{fm}^{-3}\right)$} & \multicolumn{2}{|c|}{$V_{18}$} & \multicolumn{2}{|c|}{$V_{8}^{\prime}$} & \multicolumn{2}{|c|}{$V_{6}^{\prime}$} & \multicolumn{2}{|c|}{$V_{4}^{\prime}$} \\
\hline & SM & NM & SM & NM & SM & NM & SM & NM \\
\hline 0.03 & -6.2 & 5.0 & -6.3 & 4.9 & -6.1 & 5.3 & -7.4 & 5.6 \\
\hline 0.05 & -9.0 & 6.7 & -9.3 & 6.6 & -8.8 & 7.3 & -11.2 & 7.8 \\
\hline 0.10 & -12.6 & 9.7 & -13.7 & 9.2 & -12.2 & 11.2 & -17.7 & 12.2 \\
\hline 0.17 & -15.7 & 14.0 & -18.4 & 12.4 & -15.4 & 16.7 & -26.4 & 18.2 \\
\hline 0.20 & -16.4 & 16.1 & -20.0 & 13.8 & -16.2 & 19.2 & -29.7 & 20.9 \\
\hline 0.30 & -16.0 & 25.3 & -23.7 & 19.4 & -17.0 & 28.9 & -39.2 & 31.5 \\
\hline 0.40 & -11.9 & 37.8 & -25.5 & 26.2 & -15.2 & 40.5 & -46.5 & 44.0 \\
\hline 0.50 & -4.6 & 53.6 & -26.0 & 33.9 & -11.5 & 53.9 & -51.8 & 58.4 \\
\hline
\end{tabular}

One might be tempted to attribute the small NM differences between the $V_{6}^{\prime}$ and $V_{18}$ interaction to (i) small spin-orbit components in the $N N$ interaction or (ii) small spin-orbit correlations in the medium. As we have seen, however, the partial wave expansion suggests that this is a rather fortuitous coincidence. First, both potentials are not truly phase-shift equivalent. Second, the in-medium corrections are quite different, as evidenced by the different partial wave components of the energy. All in all, our findings suggest that spin-orbit components should not be arbitrarily eliminated in $a b$ initio calculations. Extending the argument to $V_{8}^{\prime}$, one could say the same for the remaining missing operators in the interaction. Their effects are relevant for the EOS, especially at high densities, and need to be dealt with carefully.

A similar reasoning can be extended to SM. The results provided by $\tilde{V}_{6}$ (without readjusting the potential) and $V_{6}^{\prime}$ (with readjustment) are rather close in that case, indicating that the readjustment in the central $T=0, S=1$ channel is relatively small. One can therefore say that the differences between the SM $V_{6}^{\prime}$ and $V_{8}^{\prime}$ results are mainly attributable to the suppression of the spin-orbit component in the interaction itself and they give rise to rather large corrections. In other words, the suppression of the spin-orbit component does not have a small effect on the EOS. The fact that the $V_{18}$ and $V_{6}^{\prime} \mathrm{SM}$ results are relatively close at low densities is arising only from a cancellation effect in different partial waves. This can hardly be ascribed to a smallness of spin-orbit interactions or in-medium correlations. Let us point out also that the situation for the EOS is different to that of light nuclei, where $V_{6}^{\prime}, V_{8}^{\prime}$, and $V_{18}$ predict, in the framework of GFMC, somewhat similar spectra and total energies [4]. It is rather difficult to find a simple correspondence between the EOS differences presented here and the GFMC for finite nuclei. One can argue, however, that at the typically low densities corresponding to finite nuclei, the nuclear matter results provided by the different phase-shift equivalent potentials are quite close to each other. Hence, one would not expect to find large differences in finite nuclei calculations, even though they might be significant at larger densities.

\section{COMPARISON WITH OTHER MANY-BODY METHODS}

Several approaches have been devised over the decades to treat the nuclear matter many-body problem. Critical comparisons between the approaches can help us learn how correlations, produced by different pieces of the original $N N$ potentials, are treated within each scheme [14,26]. The ultimate goal of these comparisons should not be to find a "good" or a "bad" EOS, but rather to understand and quantify the differences among them. Eventually, a well-founded

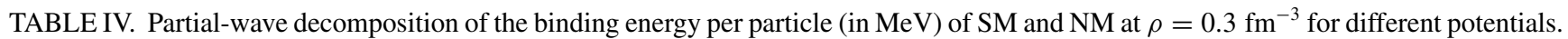
The total sum comprises partial waves up to $J=8$. The row in brackets contains the partial sum of the ${ }^{3} P_{0},{ }^{3} P_{1},{ }^{3} P F_{2}$ states.

\begin{tabular}{|c|c|c|c|c|c|c|c|c|}
\hline \multirow[t]{2}{*}{ State } & \multicolumn{4}{|c|}{ SM } & \multicolumn{4}{|c|}{$\mathrm{NM}$} \\
\hline & $V_{18}$ & $V_{8}^{\prime}$ & $V_{6}^{\prime}$ & $V_{4}^{\prime}$ & $V_{18}$ & $V_{8}^{\prime}$ & $V_{6}^{\prime}$ & $V_{4}^{\prime}$ \\
\hline \multicolumn{9}{|l|}{$\mathrm{T}=1$} \\
\hline${ }^{1} S_{0}$ & -21.9 & -23.2 & -22.4 & -22.1 & -20.4 & -22.4 & -20.8 & -20.5 \\
\hline${ }^{3} P_{0}$ & -5.0 & -5.1 & -9.1 & 0.6 & -4.2 & -4.4 & -11.4 & 1.4 \\
\hline${ }^{3} P_{1}$ & 20.2 & 19.9 & 15.6 & 1.8 & 31.5 & 30.8 & 22.6 & 4.2 \\
\hline${ }^{3} \mathrm{PF}_{2}$ & -16.5 & -16.6 & -4.2 & 3.1 & -25.4 & -25.7 & -3.6 & 7.1 \\
\hline$\left({ }^{3} P_{*}\right.$ & -1.3 & -1.8 & 2.3 & 5.5 & 1.9 & 0.7 & 7.6 & $12.7)$ \\
\hline${ }^{1} D_{2}$ & -5.9 & -6.7 & -6.7 & -6.7 & -10.0 & -12.1 & -12.1 & -12.0 \\
\hline \multicolumn{9}{|l|}{$\mathrm{T}=0$} \\
\hline${ }^{3} S D_{1}$ & -20.4 & -25.2 & -23.9 & -43.5 & & & & \\
\hline${ }^{1} P_{1}$ & 7.3 & 7.2 & 7.3 & 7.3 & & & & \\
\hline${ }^{3} D_{2}$ & -8.0 & -8.9 & -8.8 & -4.8 & & & & \\
\hline All & -16.0 & -23.7 & -17.0 & -39.2 & 25.3 & 19.4 & 28.9 & 31.5 \\
\hline
\end{tabular}



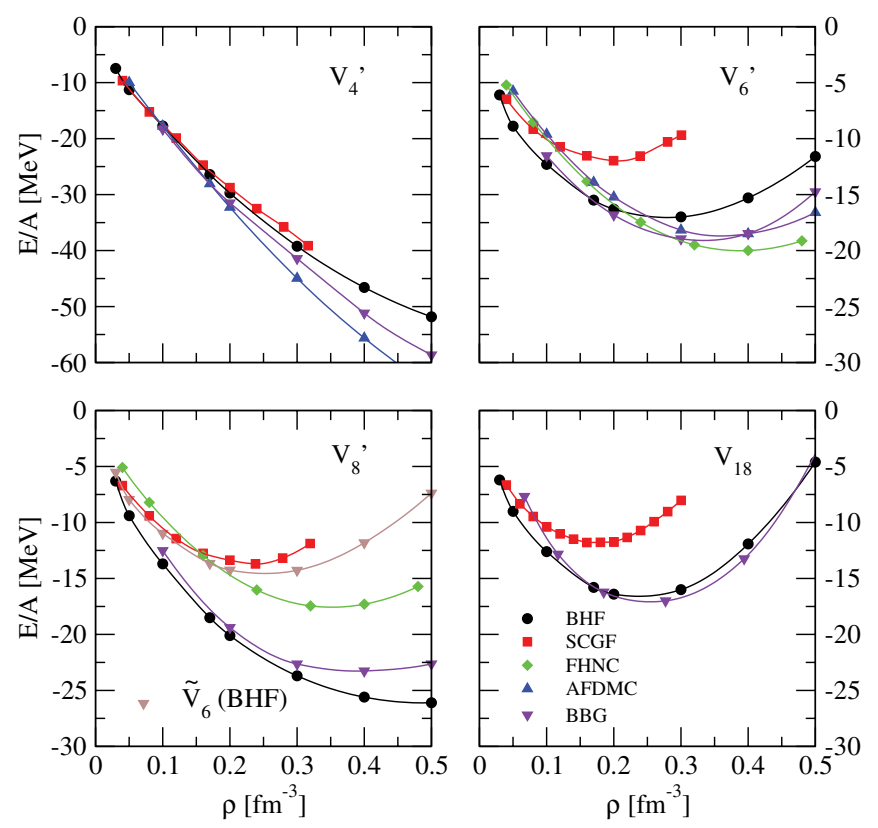

FIG. 3. (Color online) Energy per particle of SM as a function of density calculated with several many-body approaches for different Argonne potentials: $V_{4}^{\prime}$ (top left), $V_{6}^{\prime}$ (top right), $V_{8}^{\prime}$ (bottom left), and $V_{18}$ (bottom right).

comparison might also delineate the theoretical uncertainties of present-generation EOSs.

The only way to carry out a meaningful comparison between many-body approaches is by starting from the same underlying $N N$ interaction. While, in principle, one would like these to be phase-shift equivalent and as realistic as possible, some many-body approaches are currently limited by the operatorial structure of the $N N$ force. FHNC and AFDMC, in particular, are coined to treat Argonne-type interactions, involving the sum of products of local radial functions and operators. Some parts of the full $V_{18}$ interaction cannot yet be fully included in these many-body schemes. To be able to compare between many-body methods, we perform calculations with different approaches with the same family of underlying $N N$ potentials. By progressively adding components to the $N N$ force, we also hope to elucidate the role of such operators in the different many-body treatments of the EOS.

Results are summarized in Figs. 3 and 4, which represent the energy per particle as a function of density for both SM and $\mathrm{NM}$, respectively. The four panels in each figure give results for different $N N$ interactions. Within each panel, BHF results are shown as circles; SCGF as squares; FHNC as diamonds; AFDMC as up triangles; and BBG as down triangles. In the case of NM we also show available GFMC (right triangles) results [5] for the $V_{6}^{\prime}$ and $V_{8}^{\prime}$ potentials. Let us first comment the SM results and move later to NM results.

\section{A. Symmetric nuclear matter}

\section{1. $B B G$}

We start by discussing the SM results obtained with the BBG approach at the three-hole line level. Three-hole line
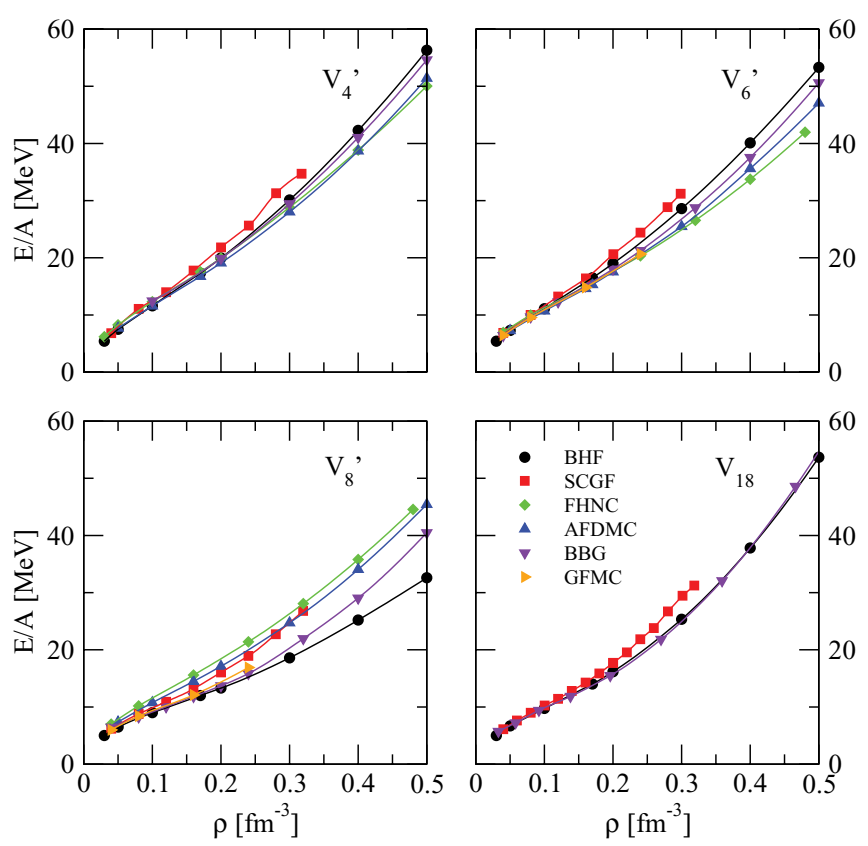

FIG. 4. (Color online) Same as Fig. 3, but for NM.

contributions are especially small when using the continuous prescription for the single-particle spectrum [10]. Using the gap prescription, the $\mathrm{BBG}$ value converges also to the same results $[10,26]$. In this case, however, the three-hole line contribution is sizable and therefore the lowest-order BHF results cannot be taken as a good estimation of the energy. We note here that the BBG results shown in this work have been calculated with the so-called $K$-matrix [7] whereas, as has been said before, the BHF ones have been obtained with the $G$-matrix. We have checked, however, that at the BHF level the differences between $G$-matrix and $K$-matrix calculations are negligible for both SM and NM and all the potentials. Only a difference of about $2 \mathrm{MeV}$ is found in the case of the $V_{8}^{\prime}$ potential for both SM and NM at the highest density considered.

We confirm the good agreement between our continuous choice BHF and the BBG calculations for all the $N N$ interactions considered. The results differ by a few $\mathrm{MeV}$ at large density, indicating, as already mentioned, a rather good convergence of the hole-line expansion. We should mention, however, that the differences between the BHF and BBG results are even smaller when the $\mathrm{BHF}$ is done with the $K$-matrix instead of the $G$-matrix. Note that the three-hole line contribution can be either attractive or repulsive, depending on the interaction under consideration. In particular, it is minimal for the most realistic potential, $V_{18}$ (bottom right panel). The BBG predictions for $V_{8}^{\prime}$ represent minor repulsive corrections to the BHF results and both lie well below the other approaches. Note, in particular, that nuclear matter saturates beyond $0.5 \mathrm{fm}^{-3}$ in the BHF case. As explained earlier, this is attributable to the partial wave contributions associated with spin-orbit and tensor forces, which are particularly strong for this potential. This is confirmed by comparison with the $\tilde{V}_{6}$ results in the same panel, in which these forces are removed and a very strong reduction of binding is observed. 
In the BHF approach the single-particle potential is calculated by means of a self-consistent procedure. If one identifies the chemical potential as the single-particle energy at the Fermi momentum, then the Hughenholtz-Van Hove theorem is violated. The theorem implies, in particular, that at saturation the chemical potential should equal the energy per particle. This type of requirement is typically violated by about $20 \mathrm{MeV}[27,28]$. However, it has to be kept in mind that in the BBG expansion the single-particle potential is an auxiliary potential, used to speed up the rate of convergence in the energy expansion. The chemical potential should be extracted from the density derivative of the energy (or free energy in general). In this case the Hughenholtz-Van Hove theorem is automatically fulfilled (see Ref. [29]).

\section{2. $S C G F$}

Another way to approach the many-body problem is through the SCGF method [9]. In this case, a diagrammatic expansion is employed to solve for the in-medium one-body propagator, rather than for the energy of the system. For infinite matter, the method is conventionally applied at the ladder approximation level. With respect to the $G$-matrix, the in-medium ladder interaction presents two major differences. First, hole-hole intermediate states are considered in addition to the typical BHF particle-particle propagators. Second, these intermediate propagators are fully dressed, that is, expressed in terms of spectral functions rather than through single-particle energies only.

At a formal level, the comparison between the BHF and the SCGF approaches is not straightforward. Even though both approaches arise from a diagrammatic expansion, the infinite subsets of diagrams considered in both approaches are not the same. Moreover, the summation procedures are also somewhat different, with the Dyson equation being used in SCGF to dress all internal propagators. Whereas the BHF formalism in the continuous choice can be derived from the ladder SCGF formalism after a series of approximations [30], this is not the case for the full $\mathrm{BBG}$ expansion.

In principle, if both BBG and SCGF were carried out to all orders, they should yield identical results. BBG theory, however, is an expansion in powers of density (or hole-lines), and the three-hole line results seem to indicate that it converges quickly [10]. The error in the SCGF expansion is more difficult to quantify, as one cannot directly compute (or even estimate) the values for the diagrams of other structural types. We show a few representative diagrams for the perturbation expansion of the energy in Fig. 5. Dashed (continuous) lines represent interactions (fermions). Up to second order, that is, diagrams (a) and (b), both approaches include the same diagrammatic contributions. The only discrepancies at this level would arise from the (potentially self-consistent) treatment of internal lines. Within the SCGF approach, this would automatically give rise to diagrams such as (c), which, in BBG theory, are considered separately. At higher orders, ring-diagram iterations, as in diagram (e) or (h), would be included in the three-hole line BBG expansion, but not in the SCGF ladder resummation. Similarly, diagram (i) represents a third-order (a)

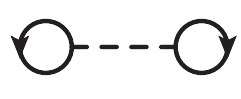

(d)

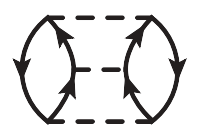

(g)

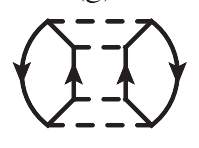

(b)

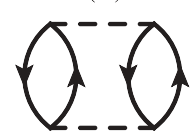

(e)

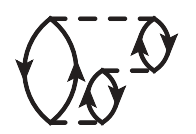

(h)

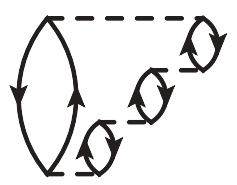

(c)

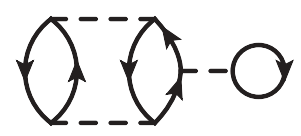

(f)

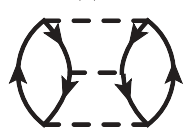

(i)

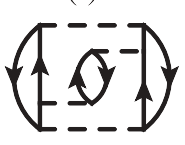

FIG. 5. Diagrams in the perturbation expansion of the total energy. Only direct terms are shown for simplicity.

bubble diagram that is not explicitly incorporated in the SCGF theory. Diagram (f) is a typical hole-hole scattering process included in SCGF and absent in BHF.

It is well established that the differences between SCGF and BHF result in an overall repulsive effect to the binding energy of both SM [30-32] and NM [11], which is mainly attributable to the inclusion of hole-hole propagation. Phase-space arguments suggest that this repulsive effect should increase with density. The dressing of intermediate propagators is capital for the thermodynamical consistency of the method. The Hugenholtz-Van Hove theorem is well fulfilled in these calculations. At the technical level, we would like to point out that the SCGF results presented here have been obtained as zero-temperature extrapolations of finite-temperature calculations. The details of the extrapolation procedure will be presented elsewhere [33]. Pure zero-temperature calculations have been obtained using different numerical techniques by the Ghent [31] and Krakow [12] groups.

Compared to BHF, the repulsive effects within the SCGF approach are quite sizable in SM, especially when the tensor component is considered. For the case of $V_{18}$ (bottom right panel in Fig. 3), for instance, the saturation point shifts from $\left[0.25 \mathrm{fm}^{-3},-16.8 \mathrm{MeV}\right]$ for BHF to $\left[0.17 \mathrm{fm}^{-3},-11.9 \mathrm{MeV}\right]$ for SCGF. While the shift seems to go towards the right saturation density, the value of the SCGF saturation energy is quite high. A similar tendency (lower saturation density, quite less binding at saturation) is also found for $V_{6}^{\prime}$ and $V_{8}^{\prime}$, in agreement with the findings of Ref. [31]. The oversimplified $V_{4}^{\prime}$ does not seem to saturate within the SCGF approach either. Note that, for this potential, the results are very close to BHF, which could suggest that, if tensor and spin-orbit correlations are not present, the many-body problem is under better control (see also the top left panel in Fig. 3).

In the SCGF approach, the total energy is usually obtained through the Koltun sum rule [9]. Even though this gives direct access to the total true kinetic and potential energies, the sum rule cannot be explicitly analyzed in terms of partial waves, as we have done for BHF. It is therefore difficult to pin down the observed differences to specific terms in the $N N$ interaction. 
Alternative ways of computing the energy, as those suggested in Ref. [12], could provide such a partial wave decomposition.

\section{3. $\mathrm{FHNC}$}

Several approaches based on the variational principle have also been devised to treat the nuclear matter problem [15]. These are rather different from the methods based on nonperturbative sums of diagrams presented earlier, but the basic principle is rather simple. The expectation value of the Hamiltonian,

$$
E=\frac{\left\langle\Psi_{T}|H| \Psi_{T}\right\rangle}{\left\langle\Psi_{T} \mid \Psi_{T}\right\rangle}
$$

in a trial wave function,

$$
\Psi_{T}=\left(\prod_{i<j} F_{i j}\right)_{\text {sym }} \phi_{\text {Fermi-Gas }},
$$

provides an upper bound to the exact energy. The trial wave function is built by means of a correlation operator,

$$
F_{i j}=\sum_{p} f^{p}\left(r_{i j}\right) O_{i j}^{p},
$$

that describes the correlations induced by the $N N$ interaction. In principle, this correlation operator should have the same operatorial structure as the interaction [see Eq. (1)]. In practice, though, this is generally not the case, because the calculation of the expectation value for the full Hamiltonian is technically very involved.

The total energy can be evaluated in a diagrammatic cluster expansion with the aid of the Fermi hypernetted chain/single operator chain (FHNC/SOC) integral equations [14], which sum Meyer-type diagrams containing up to an infinite number of nucleons. The sum is, however, incomplete, as some topologies and operatorial structures are difficult to include within infinite summations. For instance, the elementary diagrams are generally not included in FNHC calculations. Similarly, the spin-orbit correlations cannot be chained and are usually evaluated at the three-body cluster level. On top of this variational upper bound to the energy, one can add second-order perturbative corrections $\left(\Delta E^{\mathrm{CBF}}\right)$ calculated in the framework of the correlated basis function (CBF) theory [15].

The FHNC results for SM with $V_{8}^{\prime}$ and $V_{6}^{\prime}$ are presented in the corresponding panels of Fig. 3. For our comparisons, we use the FHNC calculations reported in Refs. [16,19]. There is no clear tendency with respect to our BHF reference calculations. Whereas the $V_{6}^{\prime}$ FHNC results are more attractive than the BHF calculations, a significantly repulsive behavior is observed for $V_{8}^{\prime}$. Consequently, the saturation points are quite different to those of BHF. For $V_{6}^{\prime}$, the FHNC results saturate around $\left[0.39 \mathrm{fm}^{-3},-20.1 \mathrm{MeV}\right]$, while the $V_{8}^{\prime}$ calculation saturates at $\left[0.35 \mathrm{fm}^{-3},-17.6 \mathrm{MeV}\right]$.

If the variational procedure had been exactly performed, the FHNC results should provide an upper bound to the energy per particle. However, as pointed out in Ref. [16], the uncertainties and difficulties in the treatment of the spin-orbit correlations in the FHNC formalism have hindered the inclusion of spin-orbit correlations in the wave function, that is, the underlying $N N$ interaction and correlation function are not treated on an equal footing. A widely debated question is if the variational character of FHNC results is preserved after the approximations involved in the calculational procedure. Notice also that the inclusion of 2 particle- 2 hole corrections calculated at second order in the framework of the CBF formalism would give an additional attraction to the FHNC results [18].

Contrary to what we have observed within BHF, the FHNC results seem to be quite independent of the underlying interaction. This has been attributed to the smallness of the spin-orbit components of the $N N$ interaction as well as the cancellation of spin-orbit correlations in the in-medium wave function. These arguments stem from comparisons with AFDMC results [16]. The impossibility to switch off spin-orbit correlations in BHF hinders a direct comparison with the $V_{8}^{\prime}$ FHNC calculations, where the spin-orbit correlations have been omitted. As in the case of the deuteron, to have a quantitative idea of the contribution of the spin-orbit components, we report BHF results for the $\tilde{V}_{6}$ interaction. The EOS of SM with $\tilde{V}_{6}$ is shown in the bottom left panel of Fig. 3. As explained earlier, the difference between the $V_{8}^{\prime}$ and $\tilde{V}_{6}$ results is caused only by the spin-orbit components in the original interaction. The differences turn out to be rather large and therefore this comparsion, although not conclusive, does not support the idea that switching off spin-orbit correlations produces small changes in the energy. In any case, FHNC calculations with a more elaborate treatment of the spin-orbit correlations would be highly desirable.

\section{4. $Q M C$}

Quantum Monte Carlo (QMC) methods are very successful in describing the ground state of an infinite system of bosons, such as atomic liquid ${ }^{4} \mathrm{He}$ [34], or fermions, such as liquid ${ }^{3} \mathrm{He}$ [35]. Additional efforts have allowed the extension of the QMC method to nuclear systems, which have more complicated interactions and correlation structures. However, the accuracy of QMC in its different versions, be it AFDMC [6] or GFMC [5], is limited by the fermion sign problem [36]. Up to now, the safest way to deal with this problem in nuclear systems is to keep the sample walk within a fixed nodal surface. This is an approximation and, consequently, a potential limitation of the QMC approach. AFDMC and GFMC differ in the way they treat the spin and isospin degrees of freedom. AFDMC samples the spin-isospin states by introducing Hubbard-Stratonovich auxiliary fields, whereas GFMC sums them completely. This fact limits in a drastic way the number of nucleons that GFMC can consider, generally up to about 16 . The auxiliary field strategy allows AFDMC to efficiently sample spin-isospin correlations in systems with a sufficient number of nucleons $(N=114)$ to ensure that the finite size corrections are small. A recent comparison has demonstrated that both methods give very close results for neutron drops with $N \leqslant 16$ [37].

In spite of its recent progress, the AFDMC method is still not able to work with the full $V_{18}$ potential. Technical problems, again mainly associated with the spin-orbit structure 
of the interaction and the trial wave function, are the reason for the lack of AFDMC results for SM with potentials containing spin-orbit components $\left(V_{18}\right.$ or $\left.V_{8}^{\prime}\right)$. We use the most recent version of the AFDMC results in our comparisons [20]. $V_{4}^{\prime}$ results are rather similar to our BHF reference calculation up to $0.2 \mathrm{fm}^{-3}$, but the AFDMC predictions become more attractive as density increases. The AFDMC results for SM with $V_{6}^{\prime}$, as shown in the top right panel of Fig. 3, are quite close to the FHNC predictions. Note, for instance, that AFDMC with $V_{6}^{\prime}$ saturates around $\left[0.37 \mathrm{fm}^{-3},-18.8 \mathrm{MeV}\right]$. However, the agreement between both approaches should not be taken as a final consistency check.

Let us finally comment on the results obtained with $V_{4}^{\prime}$. As an over-simplistic interaction without a tensor component, $V_{4}^{\prime}$ yields an unrealistic EOS for SM which does not saturate for any of the many-body approaches discussed here. This potential, however, can be used for benchmarking purposes. As a matter of fact, in spite of its limitations, the $V_{4}^{\prime}$ results are useful, as we find a large degree of agreement among the different many-body approaches. This confirms the long accepted notion that, in the nuclear matter case, the correlations induced by spin-orbit and tensor components of the interaction are probably the basis of the large differences when comparing different many-body methods. As a matter of fact, the antisymmetrization procedure in NM eliminates part of these components (particularly, the tensor coupling) and, as we see in the following, this results into a much closer agreement among all the many-body calculations.

\section{B. Neutron matter}

The NM results for different $N N$ interactions and manybody approaches are presented in Fig. 4. Overall, it is fair to say that the differences between the different potentials are significantly smaller for NM than for SM. As just mentioned, we attribute this similarity to the fact that, for a fully isospin-polarized system, the number of active partial waves is reduced. This is particularly true for the ${ }^{3} S D_{1}$ coupled wave, which is inactive in this system. Consequently, the differences between potentials are further reduced. Moreover, the increase of forbidden partial waves reduces the presence of tensor correlations and presumably facilitates the solution of the many-body problem.

For $V_{4}^{\prime}$ (top left panel), all reported approaches give quite similar results. This supports the idea that, when the potential is just central, a good agreement between the different manybody techniques is found. Results for low-density NM [38,39], where the interaction is mainly dominated by the ${ }^{1} S_{0}$ partial wave [40], seem to confirm this idea as it can be seen, for example, in Fig. 1 of Ref. [41]. Let us in particular stress the fact that, up to $\rho=0.3 \mathrm{fm}^{-3}$, the results of all the different approaches fall within a rather narrow window of less than $5 \mathrm{MeV}$.

When the tensor component of the force is taken into account, as in $V_{6}^{\prime}$ (see top right panel), the differences up to $\rho=0.3 \mathrm{fm}^{-3}$ are still relatively small. At this density, we find $e^{\mathrm{BHF}}=28.6 \mathrm{MeV}, e^{\mathrm{BBG}}=26.9 \mathrm{MeV}, e^{\mathrm{SCGF}}=31.2 \mathrm{MeV}$, $e^{\mathrm{AFDMC}}=25.5 \mathrm{MeV}$, and $e^{\mathrm{FHNC}}=25.0 \mathrm{MeV}$. In other words, the inclusion of the tensor component increases the uncertainty (measured as the spread predicted by these calculations) in the many-body calculations by $1-2 \mathrm{MeV}$. Note that, however, a broader discrepancy is observed at high densities, with FHNC lying below all the other approaches. In general, SCGF provides the more repulsive results. As mentioned earlier, $V_{6}^{\prime}$ and $\tilde{V}_{6}$ produce the same results for NM because the readjustment of the potential does not affect the NM partial waves.

Because the results for $V_{6}^{\prime}$ and $\tilde{V}_{6}$ are the same for NM, the differences between $V_{8}^{\prime}$ and $V_{6}^{\prime}$ give a direct measure of the spin-orbit contribution in the NM channels where it is active. The incorporation of the spin-orbit components in $V_{8}^{\prime}$ produces an overall attraction with respect to the $V_{6}^{\prime}$ results, apart from FHNC. The most attractive variation corresponds to BHF, while the SCGF, FHNC, and AFDMC approaches stay rather close to their $V_{6}^{\prime}$ counterparts at all densities. Note that also GFMC results seem to have a stronger dependence on the underlying interaction, and that they are in good agreement with the BBG ones. At $\rho=0.3 \mathrm{fm}^{-3}$, we find $e^{\mathrm{BHF}}=18.3 \mathrm{MeV}, e^{\mathrm{BBG}}=20.4 \mathrm{MeV}, e^{\mathrm{SCGF}}=24.8 \mathrm{MeV}$, $e^{\mathrm{AFDMC}}=24.7 \mathrm{MeV}$, and $e^{\mathrm{FHNC}}=26.4 \mathrm{MeV}$. The spread in these results has increased substantially compared to the simpler potentials. Again, the differences increase with density. Notice, however, that in this case the FHNC results stay above all the other methods for all densities, whereas for $V_{6}^{\prime}$ this approach provided the most attractive results.

We would like to stress once again that the spin-orbit contribution in the $N N$ interaction produces a sizable contribution for the NM BHF EOS with respect to $V_{6}^{\prime}$. This is in contrast to the small effect observed in the AFDMC or the FHNC methods. For these two methods one should notice that spin-orbit correlations (and also the tensor ones in the AFDMC case) are not included in the trial wave function, although the AFDMC allows for their full generation in the diffusion process.

The results for $V_{18}$ are only available for the three nonperturbative diagrammatic approaches, BHF, SCGF, and $\mathrm{BBG}$. The agreement between them is, in general, rather good. At $0.3 \mathrm{fm}^{-3}$, for instance, we find $e^{\mathrm{BHF}}=25.4 \mathrm{MeV}$, $e^{\mathrm{BBG}}=25.2 \mathrm{MeV}$, and $e^{\mathrm{SCGF}}=29.4 \mathrm{MeV}$. This represents a small spread in values compared with the differences we have observed in SM. One should keep in mind that these differences increase with density. Therefore, as a general remark we can conclude that in NM the change of the EOS with respect to the BHF results for both SCGF and BBG is less important than in SM. This is particularly true for the $V_{4}^{\prime}$ and $V_{6}^{\prime}$ potentials. For the $V_{8}^{\prime}$ potential, larger differences are observed between these three approaches even in NM. In all cases, the SCGF method produces the more repulsive EOS.

\section{CONCLUSIONS}

By performing calculations of the energy per particle for nuclear and NM within different many-body approaches, we have investigated the properties of the EOS as obtained with a family of frequently used Argonne potentials. The many-body approaches we have used are the BHF, the BBG approach up 
to third order in the hole-line expansion, the SCGF method, the AFDMC, and the FHNC. We have analyzed critically the origin of underlying uncertainties in these calculations. The subtraction and refitting procedure of different operatorial structures, inherent in the family of Argonne $N N$ interactions used in the present paper, has provided us with substantial insight.

At the phase shift and deuteron levels, we find that already the $V_{6}^{\prime}$ potential produces fairly large deviations relative to the original $V_{18}$ model. While at the two-body level $V_{8}^{\prime}$ is almost a clone of $V_{18}$ for $S$ and $P$ partial waves, we have found that, when included in many-body calculations, it produces relatively large differences. These discrepancies are driven by the phase-shift differences in higher partial waves as well as by off-shell effects. The $V_{8}^{\prime} N N$ interaction should be regarded critically in emulating $V_{18}$ when used at high densities in highprecision applications.

The overall infinite-matter binding energies obtained with the $V_{6}^{\prime}$ interaction are actually closer to the $V_{18}$ results, in spite of the unsatisfactory reproduction of the $P$-wave phase shifts. We believe that this agreement is, however, attributable to an artificial reshuffling of different partial wave contributions. To support this claim, we have presented the partial wave decomposition of the total energy in BHF calculations, which has indeed confirmed an overall cancellation of larger differences in the total energy. In the $T=1$ channel, this potential is not readjusted for the missing spin-orbit component, which otherwise produces fairly important contributions to the binding energy. It might well be that microscopic properties, other than the EOS, are also affected by these large discrepancies.

Finally, while the $V_{4}^{\prime}$ model is clearly unrealistic and should only be used for benchmarking purposes, we have found that it is at this level that the different many-body approaches agree more. This confirms the long-accepted hypothesis that the tensor (and spin-orbit) components of the interaction and their in-medium treatment are at the heart of most of the observed discrepancies. Overall, one needs to consider these with more attention before drawing more definite conclusions. Studies such as the one presented here help us in quantifying the uncertainties in state-of-the-art predictions of the EOS, originating either from the microscopic interaction or the many-body theory. Only a thorough understanding of these uncertainties will allow us to provide a well-founded connection between the physics of neutron stars, the fundamental strong interaction acting on its constituents, and the many-body correlations at play.

\section{ACKNOWLEDGMENTS}

We thank O. Benhar, S. Gandolfi, A. Lovato, and A. Illarionov for useful discussions. In particular, we thank S. Gandolfi and A. Lovato for providing us with the AFDMC and the FHNC results, respectively. This work is partly supported by COMPSTAR, an ESF (European Science Foundation) Research Networking Programme; by FEDER; by the initiative QREN financed by the UE/FEDER through the Programme COMPETE under the projects, PTDC/FIS/113292/2009, CERN/FP/109316/2009, CERN/FP/116366/2010 and CERN/FP/123608/2011; by the Consolider Ingenio 2010 Programme CPAN CSD2007-00042, Grant No. FIS2008-01661 from MEC and FEDER (Spain) and Grant No. 2009GR-1289 from Generalitat de Catalunya (Spain); by STFC through Grants No. (ST/I005528/1) and No. ST/J000051; and by the MIUR PRIN grant 2008KRBZTR.
[1] S. L. Shapiro and S. A. Teukolsky, Black Holes, White Dwarfs, and Neutron Stars: The Physics of Compact Objects (Wiley \& Sons, New York, 1983); N. K. Glendenning, Astronomy and Astrophysics Library, 2nd ed., Vol. 242 (Springer, New York, 2000); J. M. Lattimer and M. Prakash, Phys. Rep. 442, 109 (2007).

[2] H. Kamada, A. Nogga, W. Göckle, E. Hiyama, M. Kamimura, K. Varga, Y. Suzuki, M. Viviani, A. Kievsky, S. Rosati et al., Phys. Rev. C 64, 044001 (2001).

[3] R. B. Wiringa, V. G. J. Stoks, and R. Schiavilla, Phys. Rev. C 51, 38 (1995).

[4] R. B. Wiringa and S. C. Pieper, Phys. Rev. Lett. 89, 182501 (2002).

[5] J. Carlson, J. Morales, V. R. Pandharipande, and D. G. Ravenhall, Phys. Rev. C 68, 025802 (2003).

[6] S. Gandolfi, A. Y. Illarionov, K. E. Schmidt, F. Pederiva, and S. Fantoni, Phys. Rev. C 79, 054005 (2009); S. Gandolfi, F. Pederiva, S. Fantoni, and K. E. Schmidt, Phys. Rev. Lett. 98, 102503 (2007).

[7] M. Baldo, International Review of Nuclear Physics, Vol. 8 (World Scientific, Singapore, 1999); H.-J. Schulze, J. Cugnon, A. Lejeune, M. Baldo, and U. Lombardo, Phys. Rev. C 52, 2785 (1995).

[8] H. Müther and A. Polls, Prog. Part. Nucl. Phys. 45, 243 (2000).
[9] W. Dickhoff and D. V. Neck, Many-Body Theory Exposed, 2nd ed. (World Scientific, Singapore, 2008).

[10] H. Q. Song, M. Baldo, G. Giansiracusa, and U. Lombardo, Phys. Rev. Lett. 81, 1584 (1998); M. Baldo, G. Giansiracusa, U. Lombardo, and H. Q. Song, Phys. Lett. B 473, 1 (2000); M. Baldo, A. Fiasconaro, H. Q. Song, G. Giansiracusa, and U. Lombardo, Phys. Rev. C 65, 017303 (2001); R. Sartor, ibid. 73, 034307 (2006).

[11] A. Rios, A. Polls, and I. Vidaña, Phys. Rev. C 79, 025802 (2009); T. Frick, H. Müther, A. Rios, A. Polls, and A. Ramos, ibid. 71, 014313 (2005).

[12] V. Somà and P. Bozèk, Phys. Rev. C 74, 045809 (2006); 78, 054003 (2008).

[13] W. H. Dickhoff and C. Barbieri, Prog. Part. Nucl. Phys. 52, 377 (2004).

[14] V. R. Pandharipande and R. B. Wiringa, Rev. Mod. Phys. 51, 821 (1979).

[15] S. Fantoni and A. Fabrocini, in Microscopic Quantum ManyBody Theories and Their Applications, edited by J. Navarro and A. Polls (Springer-Verlag, Berlin, 1998), Lecture Notes in Physics, Vol. 510.

[16] A. Lovato, O. Benhar, S. Fantoni, A. Y. Illarionov, and K. E. Schmidt, Phys. Rev. C 83, 054003 (2011); R. B. Wiringa, V. Fiks, and A. Fabrocini, ibid. 38, 1010 (1988). 
[17] M. Baldo and C. Maieron, Phys. Rev. C 69, 014301 (2004).

[18] I. Bombaci, A. Fabrocini, A. Polls, and I. Vidaña, Phys. Lett. B 609, 232 (2005).

[19] A. Lovato (private communication).

[20] S. Gandolfi (private communication).

[21] Z. H. Li and H.-J. Schulze, Phys. Rev. C 78, 028801 (2008).

[22] V. G. J. Stoks, R. A. M. Klomp, M. C. M. Rentmeester, and J. J. de Swart, Phys. Rev. C 48, 792 (1993).

[23] A. Polls, H. Müther, R. Machleidt, and M. Hjorth-Jensen, Phys. Lett. B 432, 1 (1998).

[24] J. P. Jeukenne, A. Lejeune, and C. Mahaux, Phys. Rep. 25, 83 (1976).

[25] Z. H. Li, U. Lombardo, H.-J. Schulze, W. Zuo, L. W. Chen, and H. R. Ma, Phys. Rev. C 74, 047304 (2006).

[26] B. D. Day and R. B. Wiringa, Phys. Rev. C 32, 1057 (1985).

[27] N. H. Hugenholtz and W. van Hove, Physica (Ultrecht) 24, 363 (1958).

[28] P. Bozèk and P. Czerski, Eur. J. A 11, 271 (2001).
[29] M. Baldo and L. S. Ferreira, Phys. Rev. C 59, 682 (1999).

[30] T. Frick, Ph.D. thesis, University of Tübingen, 2004.

[31] Y. Dewulf, W. H. Dickhoff, D. Van Neck, E. R. Stoddard, and M. Waroquier, Phys. Rev. Lett. 90, 152501 (2003).

[32] A. Rios, Ph.D. thesis, University of Barcelona, 2007.

[33] A. Rios (unpublished).

[34] J. Boronat and J. Casulleras, Phys. Rev. B 49, 8920 (1994).

[35] J. Casulleras and J. Boronat, Phys. Rev. Lett. 84, 3121 (2000).

[36] K. E. Schmidt and M. H. Kalos, in Applications of Monte Carlo Methods in Statistical Physics, edited by K. Binder (SpringerVerlag, Berlin, 1984).

[37] S. Gandolfi, J. Carlson, and S. C. Pieper, Phys. Rev. Lett. 106, 012501 (2011).

[38] A. Gezerlis and J. Carlson, Phys. Rev. C 77, 032801(R) (2008).

[39] A. Gezerlis and J. Carlson, Phys. Rev. C 81, 025803 (2010).

[40] M. Baldo and G. F. Burgio, Rep. Prop. Phys. 75, 026301 (2012).

[41] F. J. Fattoyev and J. Piekarewicz, Phys. Rev. C 86, 015802 (2012). 